\title{
Bacteremia caused by Rothia mucilaginosa after pneumonia, in a patient with hematopoietic stem cell transplantation
}

\author{
Cihangül Bayhan, M.D..$^{a}$, Eda Karadă̆ Öncel, M.D. ${ }^{a}$, Ali B. Cengiz, M.D. ${ }^{a}$, Ayça B. Öksüz, M.D..$^{b}$ and \\ Güzide B. Aydın, M.D. ${ }^{c}$
}

\begin{abstract}
Rothia muciloginosa is a member of normal flora and rarely causes invasive disease. Immunosupressed patients have increased risk for severe infection. Here, we report a male patient with relapsed neuroblastoma hospitalized for pneumonia. After clinicalimprovement, patient's respiratory symptoms worsened again. Rothia muciloginosa was isolated from blood culture. The worsening of respiratory symptoms can be explained by hematogenous spread of bacteria. He was successfully treated with meropenem and vancomycin for 14 days. This rarely seen bacterium is known to have high mortality rates unless treated appropriately and should be considered especially in patients with malignancy due to their immunsupressed situation.

Key words: Rothia muciloginosa, bacteremia, pneumonia, neuroblastoma, transplantation.
\end{abstract}

http:/ / dx.doi.org/10.5546/aap.2016.eng.e343

\section{INTRODUCTION}

Rothia spp. generally causes dental caries and periodontal disease. Invasive disease by this agent is generally seen in immunocompromised hosts. ${ }^{1}$ Here, we report a patient with neuroblastoma who was hospitalized for pneumonia and presented $R$. muciloginosa bacteremia.

\section{CASE}

A four year and six months old male patient with relapsing neuroblastoma was admitted with cough and fever. According to his medical history,

a. Department of Pediatric Infectious Diseases, Hacettepe University Faculty of Medicine, Ankara, Turkey.

b. Department of Pediatrics, Hacettepe University Faculty of Medicine, Ankara, Turkey.

c. Department of Pediatric Oncology, Hacettepe University Faculty of Medicine \& Cancer Institute, Ankara, Turkey.

E-mail Address:

Cihangül Bayhan, M.D.: cihangulbayhan@gmail.com

Funding: None.

Conflict of interest: None.

Received: 12-30-2015

Accepted: 4-6-2016 he had developed stage IV neuroblastoma, and after receiving six courses of chemotherapy he had undergone surgical excision of right adrenal gland. Autologous hematopoietic stem cell transplantation was performed and retinoic acid therapy was administered in addition to radiotherapy. He had been in remission for approximately six months but at the age of 4 year and 4 months he had admitted because of inability to walk. A spinal magnetic resonance imaging showed dural thickening through the whole spinal cord that was compatible with central nervous system relapse. Chemotherapy and radiotherapy were started again and first course of therapy was completed one month before patient's emergency room admission. Physical examination indicated an oxygen saturation of $89 \%$ while breathing room air. Examination of his chest revealed no crackles or rhonchus. Chest radiograph revealed bilateral peribronchial infiltration consistent with bronchopneumonia (Figure 1a). Laboratory findings including total blood count and biochemical parameters were normal except a hemoglobin level of $8.6 \mathrm{~g} /$ $\mathrm{dL}$; the absolute neutrophil and lymphocyte counts were also in normal range. Ceftriaxone, clarithromycin and oseltamivir therapy was initiated. He developed tachypnea and dyspnea which increased gradually during his follow-up, and needed oxygen supply with higher flow. On the fifth day of admission chest radiograph indicated an increase in pulmonary opacities; ceftriaxone therapy changed to vancomycin and meropenem. Oseltamivir was discontinued after five days. Thorax computed tomography showed bilateral diffuse consolidation and ground-glass opacity (Figure 1b); amphotericin B, trimethoprimsulfamethoxazole and amikacin were added to therapy. Assays for cytomegalovirus, Mycoplasma pneumonia, Chlamydia pneumonia were negative. Adenovirus and human rhinovirus were detected in nasal swap sample obtained at admission by multiplex real-time reverse transcription polymerase chain reaction. The patient's condition had begun to get better from the tenth day of admission and antimicrobial therapy was stopped. 
He deteriorated suddenly on the fifteenth day accompanied by fever and his dyspnea and oxygen need were aggravated again. Chest radiograph didn't show any new findings. Complete blood count revealed a hemoglobin level of $8.4 \mathrm{~g} / \mathrm{dL}$, white blood cell of $10.6 \times 10^{9} / \mathrm{L}$, platelet count of $113 \times 10^{9} / \mathrm{L}$. There was no severe neutropenia but meropenem was started owing to underlying risk factors. Rothia mucilaginosa was isolated on blood culture; as it was susceptible to meropenem and vancomycin; vancomycin was restarted. After five days his dyspnea and oxygen requirement began to diminish. There was no growth on the blood culture obtained 48 hours after the beginning of treatment. The patient was discharged after receiving 14 days of meropenem and vancomycin therapy after a negative blood culture result.

\section{DISCUSSION}

Rothia mucilaginosa is a member of normal flora of oropharynx and upper respiratory system and not known as a frequent pathogen for immunocompetent patient., ${ }^{2,3}$ This bacterium is an opportunistic pathogen especially for immunocompromised patients and can cause bacteremia, meningitis, pneumonia, bone and joint infections, skin and soft tissue infections, endocarditis, peritonitis and prosthetic device infections. Underlying hematological malignancy, severe neutropenia, presence of indwelling catheter and mucosal damage are defined as main risk factors for invasive diseases. ${ }^{1,3-5}$ Our patient had a tendency for invasive infections, because he had many risk factors as having hematopoietic transplantation, relapsing neuroblastoma history and receiving chemotherapy and radiotherapy. He had hospitalized for pneumonia at the beginning and viral pathogens were isolated from nasal swap testing. Thus we thought he had a viral pneumonia mimicking bacterial or fungal etiology radiologically. After clinical improvement dyspnea and fever developed again. We isolated Rothia mucilaginosa from blood culture at the same time and clinical impairment was attributed to bacteremia. We hypothesized that the mucosal barrier of oropharynx was damaged during invasive procedures like aspiration or nasogastric feeding tube placement and bacteria had translocated and caused bacteremia. The second worsening of respiratory symptoms can be explained by hematogenous spread of bacteria.

FIGURE 1. 1a. Bilateral peribronchial infiltration on chest radiogram revealed at admission. 1b. Bilateral diffuse consolidation and ground-glass opacity on thorax computed tomography
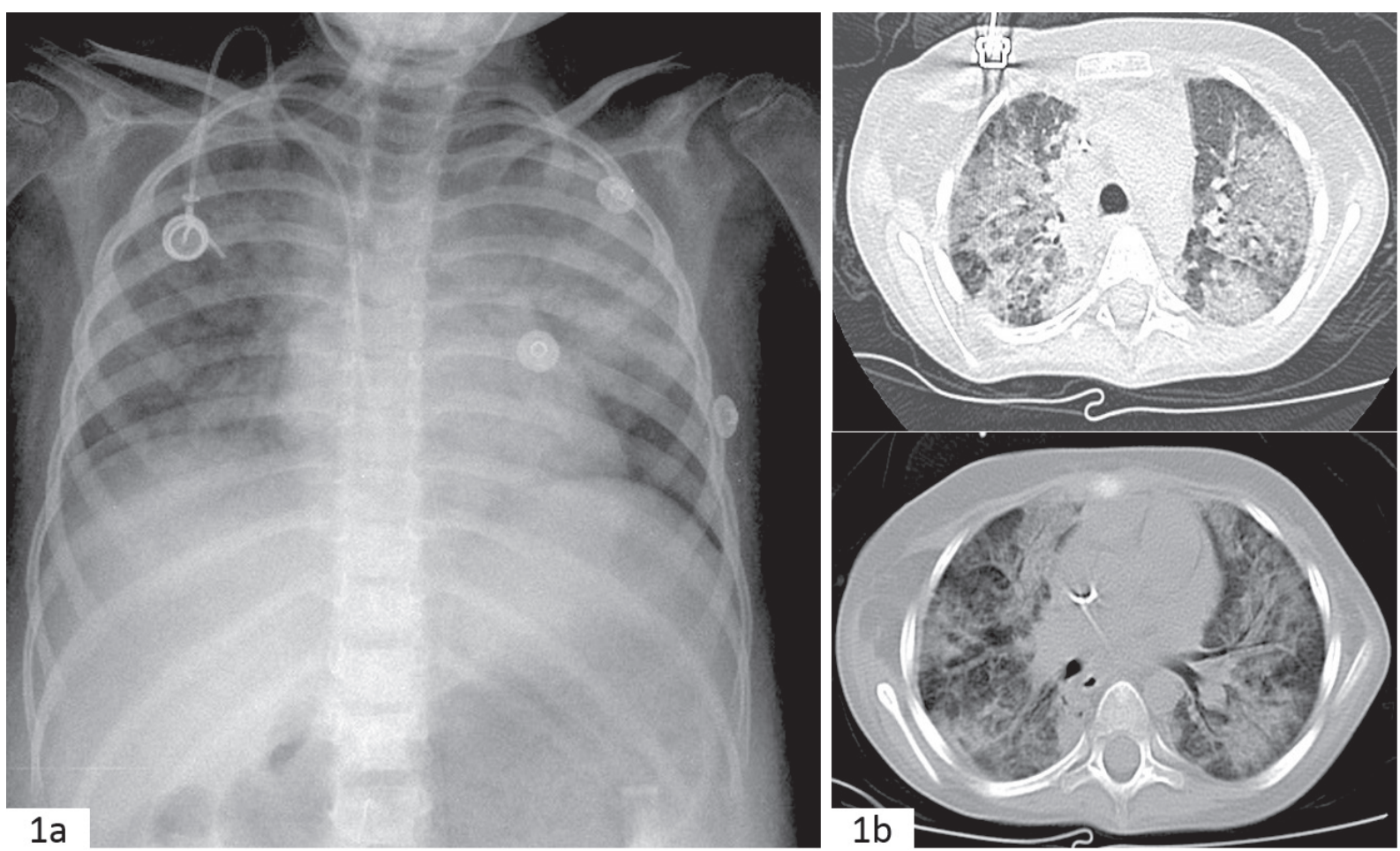
The mortality rates due to Rothia infections have varied in the literature according to age, presence of neutropenia and immunodeficiency, and type of infection. Immunocompromised patients are more susceptible to develop severe complications, including death. Chavan et al. reported 36 neutropenic patients with underlying hematologic malignancies with $R$. muciloginosa infection, and 8 of $28(28.6 \%)$ of patients with $R$. muciloginosa bacteremia died as a result of their infection. ${ }^{4}$ In another study, 10 year data about Rothia bacteremia at a single center was presented and 25 blood stream infections attributable to $R$. muciloginosa was identified including 22 neutropenic patient; one of these patient died due to the infection. ${ }^{1}$ Our patient recovered completely after antimicrobial therapy and did not demonstrate any long-term consequences of infection.

This bacterium is generally known to be susceptible to glycopeptides, third-generation cephalosporins, carbapenems and rifampicin. Penicillin, clindamycin and macrolide sensitivity is variable while resistance to quinolone and aminoglycoside is common. ${ }^{6,7}$ Treatment duration varies between different reports according to patients comorbidities; generally reported cases have antibiotic treatment for 7-21 days. In our case, Rothia muciloginosa bacteremia was detected during his pneumonia and pneumonia could be caused by this bacteria. For this reason we gave him 14 days of antibiotic therapy despite the improvement in the patient's clinical condition.

In conclusion, $R$. mucilaginosa should be considered as a likely pathogen in immunocompromised patients with suspected bacteremia, especially patients with malignancy. Rapid diagnosis and appropriate treatment are key factors in the management of this infection.

\section{REFERENCES}

1. Ramanan P, Barreto JN, Osmon DR, Tosh PK. Rothia bacteremia: a 10-year experience at Mayo Clinic, Rochester, Minnesota. J Clin Microbiol 2014;52(9):3184-9.

2. Cho EJ, Sung H,ParkSJ, Kim MN, etal. Rothia mucilaginosa pneumonia diagnosed by quantitative cultures and intracellular organisms of bronchoalveolar lavage in a lymphoma patient. Ann Lab Med 2013;33(2):145-9.

3. Morgan EA, Henrich TJ, Jarell AD, Shieh WJ, et al. Infectious granulomatous dermatitis associated with Rothia mucilaginosa bacteremia: A case report. Am J Dermatopathol 2010;32(2):175-9.

4. Chavan RS, Pannaraj PS, Luna RA, SzaboS, etal.Significant morbidity and mortality attributable to rothia mucilaginosa infections in children with hematological malignancies or following hematopoietic stem cell transplantation. Pediatr Hematol Oncol 2013;30(5):445-54.

5. Maraki S, Papadakis IS. Rothia mucilaginosa pneumonia: a literature review. Infect Dis (Lond) 2015;47(3):125-9.

6. Baeza Martinez C, Zamora Molina L, Garcia Sevila R, Gil Carbonell J, et al. Rothia mucilaginosa pneumonia in an immunocompetent patient. Arch Bronconeumol 2014;50(11):493-5.

7. Kaasch AJ, Saxler G, Seifert H. Septic arthritis due to Rothia mucilaginosa. Infection 2011;39(1):81-2. 